\title{
Antiosteoporotic effect of icariin in ovariectomized rats is mediated via the $\mathrm{Wnt} / \boldsymbol{\beta}$-catenin pathway
}

\author{
GUANGMING CHEN $^{1 *}$, CHAOPENG WANG $^{1 *}$, JIEFANG WANG $^{1}$, SUJUAN YIN ${ }^{1}$, \\ HAN GAO $^{1}$, LU XIANG ${ }^{1}$, HENGRUI LIU ${ }^{1}$, YINQUAN XIONG ${ }^{1}$, PANPAN WANG ${ }^{2}$, \\ XIAOFENG ZHU ${ }^{2}$, LI YANG $^{1}$ and RONGHUA ZHANG ${ }^{1}$ \\ ${ }^{1}$ Department of Traditional Chinese Medicine, College of Pharmacy, Jinan University; \\ ${ }^{2}$ Department of Traditional Chinese Medicine, First Affiliated Hospital of Jinan University, \\ Guangzhou, Guangdong 510632, P.R. China
}

Received May 14, 2015; Accepted April 18, 2016

DOI: $10.3892 / \mathrm{etm} .2016 .3333$

\begin{abstract}
Icariin (ICA), the main active flavonoid glucoside isolated from Herba Epimedii, has been shown to prevent postmenopausal bone loss in vitro. However, the mechanisms by which ICA prevents bone loss in vivo remain poorly understood. In the present study, the effect of ICA in an ovariectomized (OVX) rat model of osteoporosis was evaluated. Sprague-Dawley rats were divided into sham-operated and OVX groups. The OVX rats were randomly divided into five groups: OVX group (water only), Fosamax (positive) group $(5.04 \mathrm{mg} / \mathrm{kg}$, weekly, administered orally), and OVX-ICA groups $(125,250$ or $500 \mathrm{mg} / \mathrm{kg}$, daily, administered orally) and treated for 12 weeks. The 125,250 and $500 \mathrm{mg} / \mathrm{kg}$ doses of ICA were designated as low (L-ICA), medium (M-ICA) and high (H-ICA), respectively. Compared with the sham-operated group, the OVX rats had significantly decreased bone mineral density (BMD), reduced serum osteoprotegerin (OPG) and increased serum bone gla protein (BGP) concentrations. ICA significantly increased BMD, biomechanical strength, trabecular bone number and trabecular bone thickness, and reduced lumbar trabecular bone separation. Treatment with ICA also completely normalized the expression of osteoblast markers by increasing serum concentrations of OPG and BGP. Enhanced mineralization was demonstrated by increased expression of differentiation markers. Although further in vivo studies are required to investigate the efficacy of ICA in improving bone mass, this study demonstrates
\end{abstract}

Correspondence to: Professor Ronghua Zhang or Professor Li Yang, Department of Traditional Chinese Medicine, College of Pharmacy, Jinan University, 601 Huangpu Avenue West, Guangzhou, Guangdong 510632, P.R. China

E-mail: tzrh@jnu.edu.cn

E-mail: doctormonkey@126.com

*Contributed equally

Key words: icariin, Herba Epimedii, osteoporosis, mechanism, in vivo that ICA has strong osteogenic activity, inducing osteogenic differentiation and inhibiting resorption by osteoclasts. It also demonstrates an antiosteoporotic effect for ICA on the basis of BMD, biochemical markers, biomechanical tests and histopathological parameters. Compared with L-ICA and H-ICA, M-ICA was more effective and caused no liver or kidney damage.

\section{Introduction}

Osteoporosis is a disease characterized by low bone mass and deterioration of bone structure that causes bone fragility and increases the risk of fracture (1). Osteoporosis has become a major health problem that is expected to worsen with aging populations worldwide; the incidence is also rising in Asian countries. The results of a randomized controlled trial in healthy postmenopausal women, conducted by the Women's Health Initiative, has reported that the health risks of hormone replacement therapy exceed the benefits (2). Isoflavonoids are increasing considered as a promising preventive treatment for osteoporosis in a clinical setting (3). Of all the natural alternatives currently undergoing investigation, Chinese medicines have received considerable attention. Herba Epimedii (HEP), one of the foremost Chinese anti-osteoporosis formulas (4), is an important traditional Chinese herbal medicine used widely as a tonic, aphrodisiac and antirheumatic in China and Korea. Icariin (ICA; $\mathrm{C}_{33} \mathrm{H}_{40} \mathrm{O}_{15}$; molecular weight, $676.67 \mathrm{~g} / \mathrm{mol}$ ) is a flavonoid found in HEP and is considered to be the major bioactive component (5). Experiments in rats have indicated that ICA prevents bone loss and reduction in femoral strength induced by ovariectomy (OVX) $(6,7)$.

As yet, the molecular mechanism of action of ICA in the prevention of osteoporosis has not been investigated in vivo. As a step towards addressing this, the present study aimed to evaluate the effects of ICA in preventing osteoporosis and ameliorating bone loss in rats subjected to OVX. The study also attempted to elucidate the molecular mechanism of action of ICA in OVX model rats. In particular, the $\beta$-catenin pathway, including low-density lipoprotein receptor-related

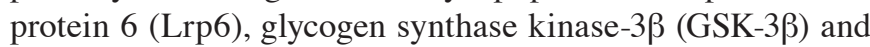
runt-related transcription factor 2 (Runx2) was investigated. 


\section{Materials and methods}

Animals and diets. A total of 84 specific-pathogen-free female Sprague-Dawley rats, 3 months of age, were purchased (Animal Center of Guangdong Province, Guangzhou, China) and acclimated to laboratory conditions for 2 weeks prior to the study. The animals were housed in an air-conditioned room at constant temperature $\left(23 \pm 2^{\circ} \mathrm{C}\right)$ and humidity (45-50\%), with $12 \mathrm{~h} / 12 \mathrm{~h}$ light-dark illumination cycles. The animals were fed in the Laboratory Animal Management Center of Jinan University Medical College (Guangzhou, China). At the beginning of the study, the animals were 3 months of age with an average weight of $250 \pm 20 \mathrm{~g}$. The present study was approved by the Animal Ethics Committee of Jinan University (Guangzhou, China).

Chemicals. ICA (EPE-120215; purity, 98\%) was purchased from Changsha Nutramax Inc. (Changsha, China). Reagent kits for the measurement of serum osteoprotegerin (OPG) and bone Gla protein (BGP) were purchased from Cloud-Clone Corp., (Houston, TX, USA). Reagent kits for the measurement of serum alanine aminotransferase (ALT) and aspartate aminotransferase (AST) were purchased from Sekisui Medical Co., Ltd. (Tokyo, Japan). Reagent kits for the measurement of blood urea nitrogen (BUN) and creatinine (Cr) were obtained from Mike Biotechnology Co., Ltd. (Sichuan, China).

Ovariectomy and administration of ICA. The rats were randomly divided into two groups: The sham-operated group $(n=14)$ and the OVX group $(n=70)$. Surgery was performed under intraperitoneal pentobarbital sodium $(0.15 \mathrm{ml} / 100 \mathrm{~g})$ anesthesia. Bilateral ovariectomies were performed from the midline with an abdominal skin incision $\sim 1.5 \mathrm{~cm}$ long. The sham-operated rats were subjected to surgery exposing the ovaries, but these were not removed. After 3 months, the bone mineral density (BMD) of the lumbar vertebrae and femurs of all rats was determined using dual-energy X-ray absorptiometry (DEXA; Lunar iDXA; GE Healthcare Bio-Sciences, Pittsburgh, PA, USA)) to evaluate whether the model had been successfully established.

Once it had been determined that the model was a success, the OVX model rats were randomly divided into five groups, each comprising 14 animals: OVX group, treated with $0.2 \mathrm{ml} / 100 \mathrm{~g}$ double distilled water, daily, administered orally; positive group treated with $5.04 \mathrm{mg} / \mathrm{kg}$ Fosamax (alendronate sodium; Merck Sharp \& Dohme, Kenilworth, NJ, USA) weekly, administered orally; and three OVX-ICA groups, treated with 125,250 or $500 \mathrm{mg} / \mathrm{kg}$ ICA, daily, administered orally. The treatments were administered for 12 weeks. The 125,250 and $500 \mathrm{mg} / \mathrm{kg}$ doses of ICA were designated as low (L-ICA), medium (M-ICA) and high (H-ICA), respectively.

After 12 weeks of treatment, blood samples were withdrawn from rats in all groups using the abdominal aorta method to assess biochemical parameters. The animals were then sacrificed using pentobarbital sodium, and the femurs, tibias and lumbar vertebrae were dissected and stored in a freezer at $-80^{\circ} \mathrm{C}$.

Measurement of BMD. BMD $\left(\mathrm{g} / \mathrm{cm}^{2}\right)$ is log-normally distributed for all individuals at the femoral neck but normally distributed at the trochanter in men and postmenopausal women (8); therefore, the assessment of BMD can be used to evaluate osteoporosis in the clinic. Following 12 weeks of treatment, the BMD $\left(\mathrm{g} / \mathrm{cm}^{2}\right)$ was assessed using DEXA in the small animal scan mode with the rats under intraperitoneal pentobarbital sodium $(0.15 \mathrm{ml} / 100 \mathrm{~g})$ anesthesia. The BMD of the fourth and fifth lumbar vertebrae and the femur was measured in all rats.

Biomechanical parameters. The freshly isolated right femur and the fifth lumbar vertebra were assessed using the three-point bending test and the compression test (9). Measurements were carried out using a precision biological materials testing system (MTS MINI 858; MTS Systems Corp., Eden Prairie, MN, USA) and the readings were recorded in Newtons.

Assessment of bone microstructure using micro-computed tomography $(C T)$. In recent years, high resolution micro-CT has become feasible for in vitro or in vivo evaluation of bone architecture. In comparison with other imaging techniques, micro-CT is more effective for detecting early bone changes that allow fracture prediction and assessment of potential antiosteoporotic agents (10). The freshly isolated fourth lumbar vertebrae were assessed using micro-CT ( $\mu$ CT 80; Scanco Medical AG, Brüttisellen, Switzerland). The fourth lumbar vertebrae were placed in the micro-CT scanning device and scanned images were obtained from different sections of the same specimen. Each plane was $20 \mu \mathrm{m}$. Morphometric parameters, including trabecular thickness (Tb.Th, $\mu \mathrm{m}$ ), trabecular number (Tb.N, 1/mm) and trabecular separation (Tb.Sp, $\mu \mathrm{m}$ ) were measured using MedProject 4.1 software and bone tissue was reconstructed through $3 \mathrm{D}$ images.

Serum parameters. ALT, AST, BUN and Cr were measured using an automatic analyzer (Ciba-Corning 550; Ciba Corning Diagnostics, East Walpole, MA, USA) with the appropriate diagnostic reagent kit. Levels of OPG and BGP were determined using an enzyme-linked immunosorbent assay reagent kit (Cloud-Clone Corp.).

Histopathology of femur bone. Femur samples were dehydrated through a graded ethanol series, defatted with chloroform and subsequently hydrated and decalcified for 1-2 weeks in formic acid. Following this, the decalcified tissues were dehydrated using increasing concentrations of ethanol, embedded in paraffin and cut into 4-mm-thick sections along the femoral axis in the sagittal plane. Serial sections were mounted on slides and dried overnight prior to staining with hematoxylin and eosin (H\&E) to observe pathological changes in rats from each group. Images of the distracted zones were captured under $\mathrm{x} 40$ magnification using a light microscope (CFX41; Olympus Corp., Tokyo, Japan).

RNA isolation, cDNA synthesis and reverse transcription-quantitative polymerase chain reaction (RT-qPCR) analysis. Femur samples were ground in liquid nitrogen using a mortar and pestle to avoid RNA degradation. Extraction of total RNA was performed using TRIzol according to the manufacturer's protocol (Invitrogen; Thermo Fisher Scientific, Inc., Waltham, MA, USA). The purified RNA sample was 
Table I. Primer sequences used in the reverse transcription-quantitative polymerase chain reaction assay.

\begin{tabular}{lll}
\hline Gene & \multicolumn{1}{c}{ Forward sequence $\left(5^{\prime}\right.$ to $\left.3^{\prime}\right)$} & Reverse sequence $\left(5^{\prime}\right.$ to $\left.3^{\prime}\right)$ \\
\hline$\beta$-catenin & ACTCTAGTGCAGCTTCTGGGTTCTG & CTCGGTAATGTCCTCCCTGTCA \\
GSK-3 $\beta$ & ACACCTGCCCTCTTCAACTTTACC & ATTGGTCTGTCCACGGTCTCCA \\
Lrp6 & GGAAGCCTAAGATTGACAGAGCG & GTTGAGTCCGAGCATATTTGAAG \\
Runx2 & AGCGGACGAGGCAACAGTTT & CCTAAATCACTGAGGCGGTCAG \\
GAPDH & CAACGGGAAACCCATCACCA & ACGCCAGTAGACTCCACGACAT
\end{tabular}

GSK-3 $\beta$, glycogen synthase kinase-3 $\beta$; Lrp6, low-density lipoprotein receptor-related protein 6; Runx2, runt-related transcription factor 2; GAPDH, glyceraldehyde 3-phosphate dehydrogenase.

Table II. Effects of OVX and ICA on BMD 12 weeks after surgery $\left(\mathrm{g} / \mathrm{cm}^{2}\right)$.

A, Effect of OVX on BMD

\begin{tabular}{lccr}
\hline Group & Lumbar vertebrae & Right femur & Left femur \\
\hline Sham & $0.285 \pm 0.009$ & $0.340 \pm 0.020$ & $0.310 \pm 0.013$ \\
OVX & $0.212 \pm 0.006^{\mathrm{a}}$ & $0.262 \pm 0.006^{\mathrm{a}}$ & $0.247 \pm 005^{\mathrm{a}}$ \\
\hline
\end{tabular}

B, Effect of ICA on BMD in OVX rats

\begin{tabular}{lcccc}
\hline Group & Lumbar vertebrae & Right femur & Left femur & Whole body \\
\hline Sham & $0.266 \pm 0.026$ & $0.274 \pm 0.034$ & $0.255 \pm 0.030$ & $0.184 \pm 0.003$ \\
OVX & $0.196 \pm 0.009^{\mathrm{b}}$ & $0.185 \pm 0.010^{\mathrm{b}}$ & $0.194 \pm 0.010^{\mathrm{b}}$ & $0.174 \pm 0.007^{\mathrm{a}}$ \\
Positive & $0.261 \pm 0.024^{\mathrm{c}}$ & $0.263 \pm 0.038^{\mathrm{c}}$ & $0.260 \pm 0.045^{\mathrm{c}}$ & $0.176 \pm 0.005^{\mathrm{a}}$ \\
L-ICA & $0.226 \pm 0.037$ & $0.257 \pm 0.020^{\mathrm{c}}$ & $0.249 \pm 0.030^{\mathrm{d}}$ & $0.173 \pm 0.006^{\mathrm{a}}$ \\
M-ICA & $0.240 \pm 0.029^{\mathrm{c}}$ & $0.254 \pm 0.017^{\mathrm{c}}$ & $0.285 \pm 0.029^{\mathrm{c}}$ & $0.174 \pm 0.006^{\mathrm{a}}$ \\
H-ICA & $0.218 \pm 0.027^{\mathrm{a}}$ & $0.243 \pm 0.016^{\mathrm{c}}$ & $0.251 \pm 0.044^{\mathrm{d}}$ & $0.171 \pm 0.007^{\mathrm{a}}$ \\
\hline
\end{tabular}

As shown in A, the BMD of the fourth and fifth lumbar vertebrae was reduced by $25.6 \%$ following OVX. As shown in B, administration of M-ICA to OVX rats for 12 weeks significantly restored BMD. ${ }^{\mathrm{P}} \mathrm{P}<0.05,{ }^{\mathrm{b}} \mathrm{P}<0.01 \mathrm{vs}$. sham; ${ }^{\mathrm{C}} \mathrm{P}<0.01,{ }^{\mathrm{d}} \mathrm{P}<0.05$ vs. OVX. OVX, ovariectomy; ICA, icariin; BMD, bone mineral density; Sham, sham-operated; Positive, treated with alendronate sodium; L-ICA, low dose ICA (125 mg/kg/day); M-ICA, medium dose ICA (250 mg/kg/day); H-ICA, high dose ICA (500 mg/kg/day).

incubated with QuantiNova gDNA Removal Mix (Qiagen $\mathrm{GmbH}$, Hilden, Germany) at $45^{\circ} \mathrm{C}$ for $2 \mathrm{~min}$. Following genomic DNA removal, $1 \mu \mathrm{g}$ RNA was reverse transcribed to cDNA using a QuantiNova reverse transcription kit (Qiagen $\mathrm{GmbH}$ ). The total reaction volume of $20 \mu \mathrm{l}$ contained $15 \mu \mathrm{l}$ RNA, $1 \mu \mathrm{l}$ QuantiNova Reverse Transcription Enzyme and $4 \mu \mathrm{l}$ QuantiNova Reverse Transcription Mix. Following a 3 min primer annealing step at $25^{\circ} \mathrm{C}$, the reaction was performed at $45^{\circ} \mathrm{C}$ for $10 \mathrm{~min}$ and subsequently inactivated at $85^{\circ} \mathrm{C}$. qPCR analysis was performed using a LightCycler 480 instrument (Roche Diagnostics GmbH, Mannheim, Germany) with a total reaction volume of $50 \mu \mathrm{l}$ comprising $25 \mu 1$ SYBR green PCR master mix (Takara Bio, Inc., Otsu, Japan), $5 \mu \mathrm{l}$ cDNA, $2 \mu 1$ each primer and $16 \mu 1$ RNAase-free water. The qPCR analysis was performed with an initial incubation temperature of $95.0^{\circ} \mathrm{C}$ for $5 \mathrm{~min}$, followed by a two-step cycling protocol, with a denaturation step at $95^{\circ} \mathrm{C}$ for $5 \mathrm{sec}$ and a combined annealing/extension step at $60^{\circ} \mathrm{C}$ for
$30 \mathrm{sec}$, carried out for 40 cycles. Melting curve analysis was performed to determine the product specificity. The respective primer sequences are shown in Table I. As a control gene, glyceraldehyde 3-phosphate dehydrogenase (GAPDH) exhibits stable and rich expression, therefore it was chosen to assess the performance of the machine via the melting and amplification curves. Expression levels were normalized against GAPDH according to the $2^{-\triangle \Delta C q}$ method (11).

Western blot analysis. A cell lysis method was used to extract total protein from the rat femoral heads and the bicinchoninic acid method was used to measure the total protein concentration in each group. Samples were run on a $12 \%$ sodium dodecyl sulfate-polyacrylamide gel electrophoresis gel and transfered onto nitrocellulose membranes. Then, immunoblotting was assayed using rabbit anti- $\beta$-catenin $(1: 2,000 ; 9562)$ and GAPDH $(1: 1,000 ; 2118$; both Cell Signaling Technology, Inc., Danvers, MA, USA) polyclonal antibodies at $4^{\circ} \mathrm{C}$ for 
Table III. Effect of ICA on liver and kidney function in OVX rats after 12 weeks.

\begin{tabular}{lllrr}
\hline Group & BUN & Cr & ALT & AST \\
\hline Sham & $4.84 \pm 0.69$ & $35.20 \pm 8.64$ & $51.00 \pm 12.98^{\mathrm{a}}$ & $81.75 \pm 18.25$ \\
OVX & $6.64 \pm 1.85^{\mathrm{b}}$ & $32.00 \pm 13.74$ & $87.80 \pm 11.90^{\mathrm{b}}$ & $121.40 \pm 71.90$ \\
Positive & $6.84 \pm 1.19$ & $35.78 \pm 6.53$ & $53.00 \pm 9.68^{\mathrm{a}}$ & $101 . \pm 7.49$ \\
L-ICA & $5.40 \pm 1.04$ & $36.00 \pm 4.76$ & $68.80 \pm 31.59$ & $104.40 \pm 41.4$ \\
M-ICA & $5.19 \pm 0.73^{\mathrm{a}}$ & $37.17 \pm 3.37$ & $41.86 \pm 13.20^{\mathrm{a}}$ & $75.71 \pm 8.28$ \\
H-ICA & $6.05 \pm 0.68^{\mathrm{b}}$ & $41.14 \pm 5.70^{\mathrm{a}}$ & $59.59 \pm 29.97$ & $63.66 \pm 29.08^{\mathrm{a}}$
\end{tabular}

Compared with the untreated OVX group, administration of M-ICA for 12 weeks restored BUN and ALT to normal levels (both P<0.05). Liver and kidney function index tests show that liver and kidney damage were lower in the M-ICA group than in the sham group. Levels of BUN in the H-ICA group were significantly increased compared with those in the sham-operated group. ${ }^{\mathrm{a}} \mathrm{P}<0.05$ vs. OVX; ${ }^{\mathrm{b}} \mathrm{P}<0.05$ vs. sham. OVX, ovariectomy; ICA, icariin; BUN, blood urea nitrogen; Cr, creatinine; ALT, alanine aminotransferase; AST, aspartate aminotransferase; Sham, sham-operated; Positive, treated with alendronate sodium; L-ICA, low dose ICA (125 mg/kg/day); M-ICA, medium dose ICA (250 mg/kg/day); $\mathrm{H}-\mathrm{ICA}$, high dose ICA (500 mg/kg/day).

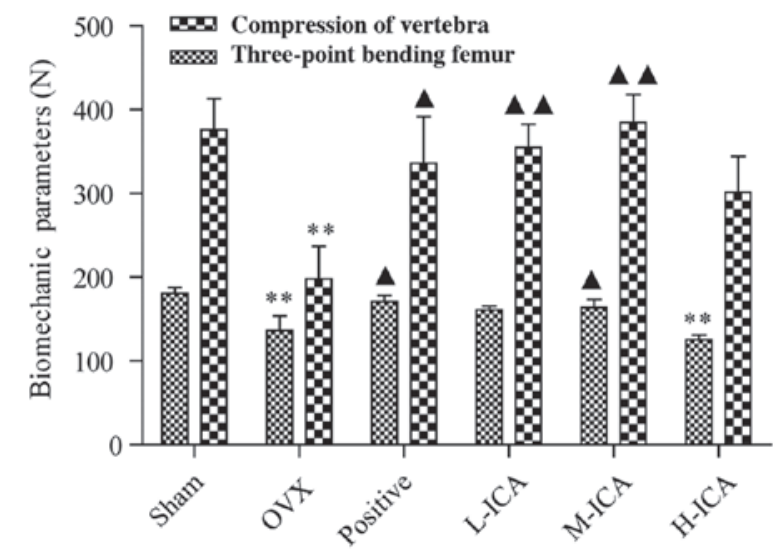

Figure 1. Three-point bending and compression tests to assess bone strength of the femur and vertebra. ${ }^{* *} \mathrm{P}<0.01$ vs. sham-operated; ${ }^{\mathbf{\Delta}} \mathrm{P}<0.05$, ${ }^{\wedge} \mathbf{A} \mathrm{P}<0.01$ vs. OVX. OVX, ovariectomy; ICA, icariin; Sham, sham-operated; Positive, treated with alendronate sodium; L-ICA, low dose ICA (125 mg/kg/day); M-ICA, medium dose ICA (250 mg/kg/day); H-ICA, high dose ICA (500 mg/kg/day).

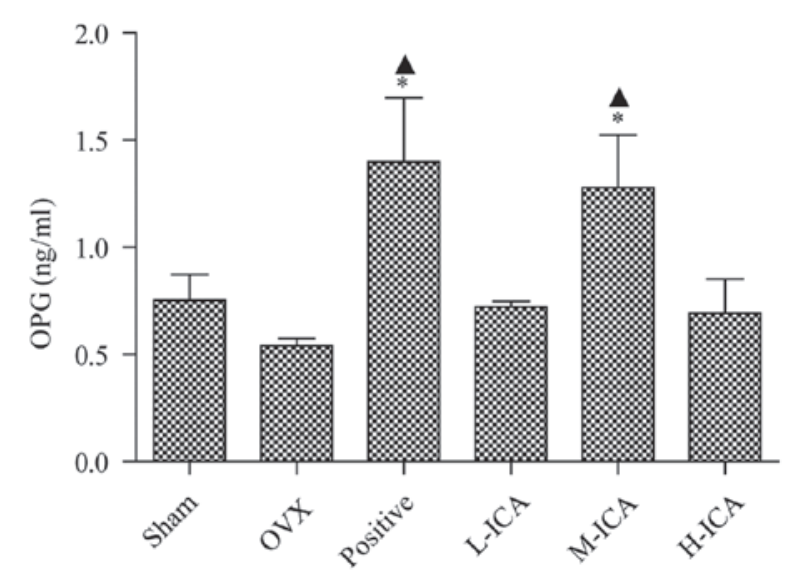

Figure 2. OPG levels in the six groups. As compared with the sham-operated group, expression of OPG was decreased in the OVX group and OPG levels were significantly increased in the positive and M-ICA groups. ${ }^{*} \mathrm{P}<0.05$ vs sham-operated; ${ }^{\wedge} \mathrm{P}<0.05$ vs. OVX. OPG, osteoprotegerin; OVX, ovariectomy; ICA, icariin; Sham, sham-operated; Positive, treated with alendronate sodium; L-ICA, low dose ICA (125 mg/kg/day); M-ICA, medium dose ICA (250 mg/kg/day); H-ICA, high dose ICA (500 mg/kg/day).

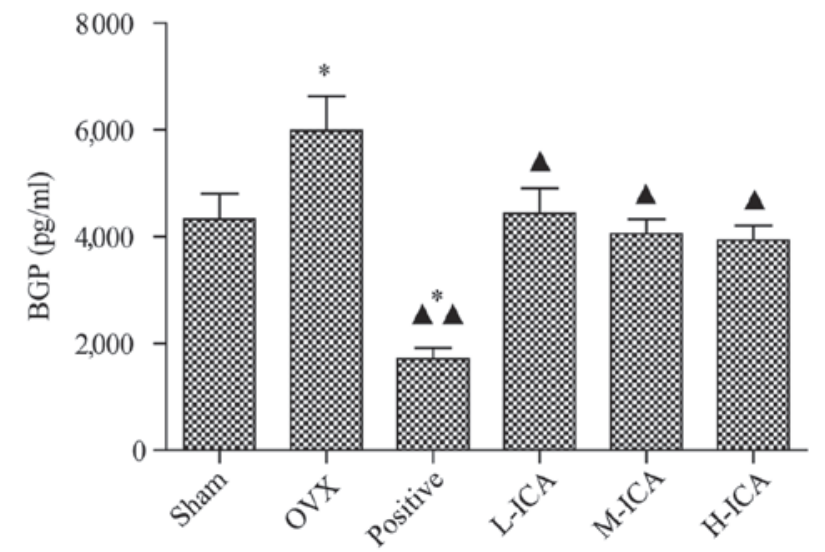

Figure 3. BGP levels in the six groups. Compared with the sham-operated group, BGP levels in the OVX group increased significantly $(\mathrm{P}<0.05)$. Compared with OVX group, levels of BGP in the positive group $(\mathrm{P}<0.01)$, and the L-ICA, M-ICA and H-ICA groups were significantly reduced $(\mathrm{P}<0.05)$. ${ }^{*} \mathrm{P}<0.05$ vs. sham-operated; ${ }^{\boldsymbol{\Delta}} \mathrm{P}<0.05$ and ${ }^{\boldsymbol{\Delta}}{ }^{\boldsymbol{A}} \mathrm{P}<0.01$ vs. OVX BGP, bone Gla protein; OVX, ovariectomy; ICA, icariin; Sham, sham-operated; Positive, treated with alendronate sodium; L-ICA, low dose ICA (125 mg/kg/day); M-ICA, medium dose ICA (250 mg/kg/day); H-ICA, high dose ICA $(500 \mathrm{mg} / \mathrm{kg} /$ day $)$.

$12 \mathrm{~h}$. Blots were visualized using horseradish peroxidase (HRP)-conjugated anti-rabbit secondary antibody (1:2,000; 7074S; Cell Signaling Technology, Inc.) at room temperature for $1 \mathrm{~h}$, with ECL Western Blotting Substrate (EMD Millipore, Billerica, MA, USA) as the substrate of HRP. Bands were detected and analyzed using ImageJ software (version 1.48; National Institutes of Health, Bethesda, MA, USA)

Statistical analysis. Statistical analyses were performed using SPSS software (version 19.0: IBM SPSS, Armonk, NY, USA). Data are presented as mean value \pm standard deviation (SD). The effects of ICA in OVX rats were analyzed by one-way analysis of variance followed by Dunnett's test (post hoc analysis). Differences between two groups were analyzed using the independent t-test or Mann-Whitney test (two-tailed), as appropriate. $\mathrm{P}<0.05$ was considered to indicate a statistically significant difference. 
A

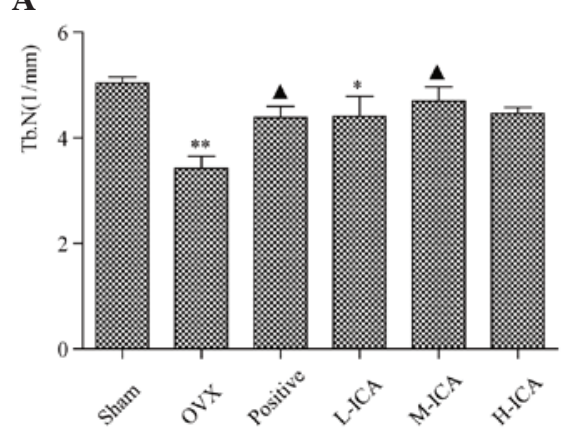

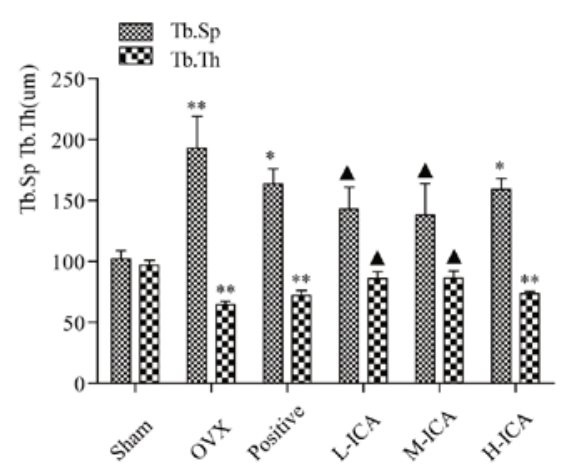

B
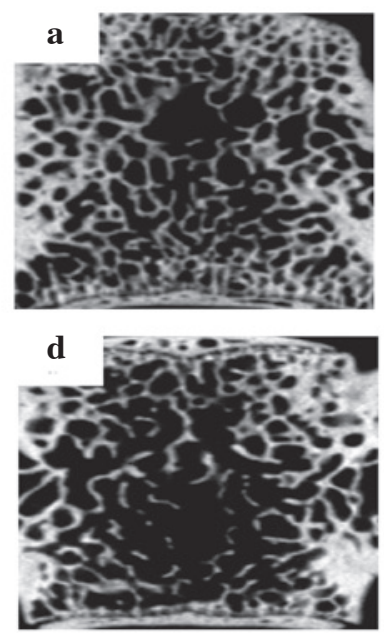
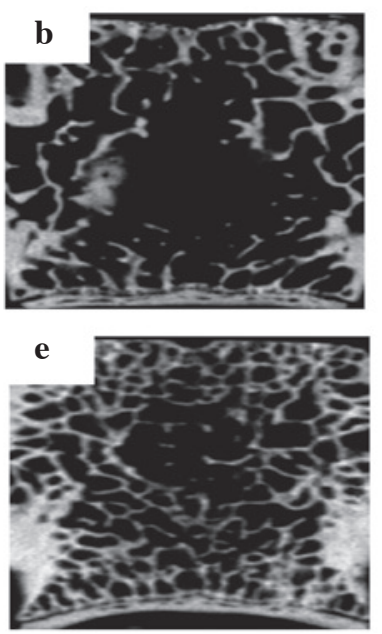
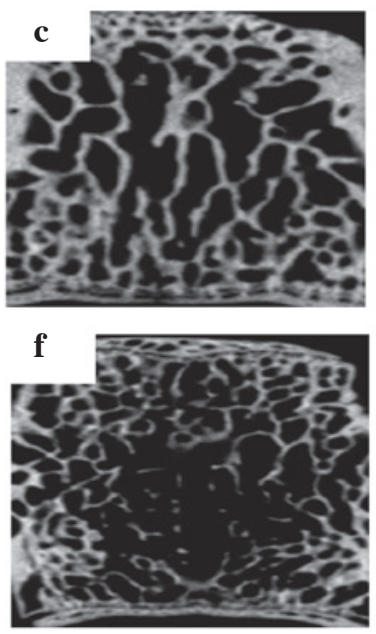

Figure 4. Morphometric analysis. (A) Tb.N and Tb.Th decreased significantly in the OVX group compared with the sham group ( $\mathrm{P}<0.01)$. Compared with the OVX group, the M-ICA group had significantly increased Tb.N and Tb.Th $(\mathrm{P}<0.05)$, while Tb.Sp decreased significantly $(\mathrm{P}<0.05)$, whereas in the L-ICA group, Tb.Th increased significantly and Tb.Sp decreased significantly $(\mathrm{P}<0.05)$. ${ }^{*} \mathrm{P}<0.05,{ }^{* *} \mathrm{P}<0.01$ vs. sham-operated; ${ }^{\mathbf{}} \mathrm{P}<0.05$ vs. OVX. (B) Micro-computed tomography analysis for the (a) sham, (b) OVX, (c) positive, (d) L-ICA, (e) M-ICA and (f) H-ICA groups. In comparison with the sham group, the bone trabecula is loose, fractured and exhibits loss in the OVX group. Following oral administration of ICA, the number of bone trabecula was clearly increased and the bone trabecula was more densely concatenated than that in the OVX group. Tb.N, trabecular number; Tb.Th, trabecular thickness; Tb.Sp, trabecular separation; OVX, ovariectomy; ICA, icariin; Sham, sham-operated; Positive, treated with alendronate sodium; L-ICA, low dose ICA (125 mg/kg/day); M-ICA, medium dose ICA (250 mg/kg/day); H-ICA, high dose ICA (500 mg/kg/day).

\section{Results}

Effects of OVX and ICA on BMD. The BMD of OVX rats was significantly lower than that of sham-operated rats $(\mathrm{P}<0.05)$. Following 12 weeks of treatment, the BMD of the OVX model rats treated with ICA was significantly higher than that of OVX rats (Table II). Compared with the OVX group, the BMD of the lumbar vertebrae, right femur and left femur significantly increased $(\mathrm{P}<0.01)$ in the positive group; whereas the BMD of the right and left femurs significantly increased $(\mathrm{P}<0.01$ and $\mathrm{P}<0.05$, respectively) in the L-ICA group. In the M-ICA group, the lumbar vertebrae, right femur and left femur BMD significantly increased $(\mathrm{P}<0.01)$; and, in the H-ICA group, the BMD of the right and left femurs significantly increased $(\mathrm{P}<0.01$ and $\mathrm{P}<0.05$, respectively). The BMD of the OVX model rats treated with ICA were significantly increased, as compared with the OVX rats. The M-ICA was most effective in comparison with the positive group (Table II).

Biomechanical tests. The bone strengths of the femurs and vertebrae, which were assessed by the three-point bending test and the compression test, respectively, decreased significantly in the OVX group compared with the sham-operated group $(\mathrm{P}<0.01)$. Following the administration of ICA to the OVX model rats, the biomechanical strength increased significantly and was comparable to that of the sham-operated group (Fig. 1). Following 12 weeks of treatment, as compared with the OVX group, vertebral and femoral strength significantly increased $(\mathrm{P}<0.05)$ in the positive group, vertebral strength increased significantly $(\mathrm{P}<0.01)$ in the L-ICA group, and femoral and vertebral strength increased significantly $(\mathrm{P}<0.05$ and $\mathrm{P}<0.01$, respectively) in the M-ICA group. M-ICA was most effective in comparison with the positive group.

Effect of OVX and ICA on OPG and BGP serum biochemical indicators. OPG levels in sham-operated and OVX rats were $0.752 \pm 0.121$ and $0.541 \pm 0.034 \mathrm{ng} / \mathrm{ml}$, respectively. OPG levels were significantly increased $(\mathrm{P}<0.05)$ to $1.28 \pm 0.244 \mathrm{ng} / \mathrm{ml}$ following treatment with M-ICA (Fig. 2). BGP levels in the sham-operated group were $4,328 \pm 0.476 \mathrm{ng} / \mathrm{ml}$. As compared with the sham-operated group, BGP levels were significantly increased $(\mathrm{P}<0.05)$ to $5,983 \pm 0.645 \mathrm{ng} / \mathrm{ml}$ in the OVX group; however, they were significantly decreased $(\mathrm{P}<0.05)$ to $1.713 \pm 0.202 \mathrm{ng} / \mathrm{ml}$ in the positive group. As compared with the OVX group, BGP levels in the positive, L-ICA, M-ICA and H-ICA groups were significantly decreased $(\mathrm{P}<0.05)$ to $1.713 \pm 0.202,4.436 \pm 0.477,4.055 \pm 0.273$ and $3.939 \pm 0.269 \mathrm{ng} / \mathrm{ml}$, respectively (Fig. 3). 
A

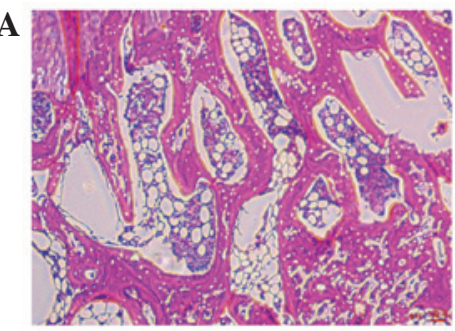

D

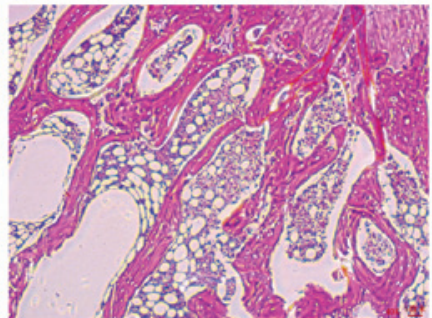

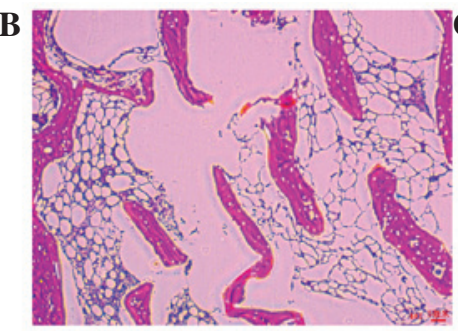
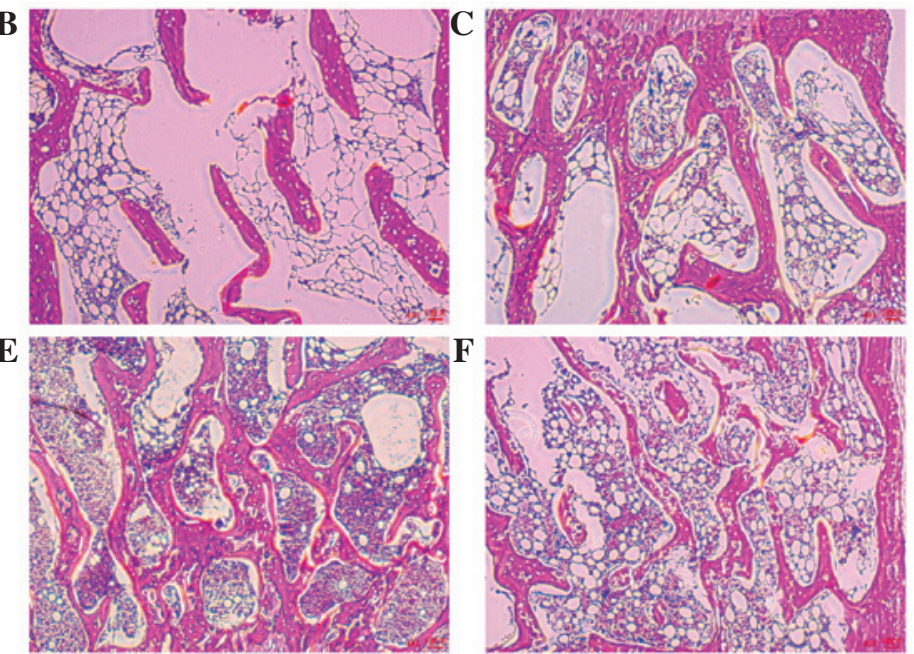

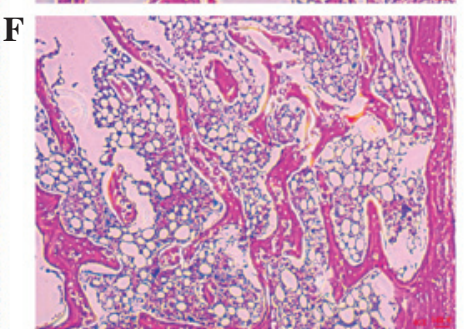

Figure 5. Histopathology of femurs from the six groups. (A) Sham, (B) OVX (C) positive, (D) L-ICA, (E) M-ICA and (F) H-ICA groups. Hematoxylin and eosin staining (magnification, x40). OVX, ovariectomy; ICA, icariin; Sham, sham-operated; Positive, treated with alendronate sodium; L-ICA, low dose ICA (125 mg/kg/day); M-ICA, medium dose ICA ( $250 \mathrm{mg} / \mathrm{kg} /$ day); H-ICA, high dose ICA (500 mg/kg/day)..ask for letters to be removed.

A

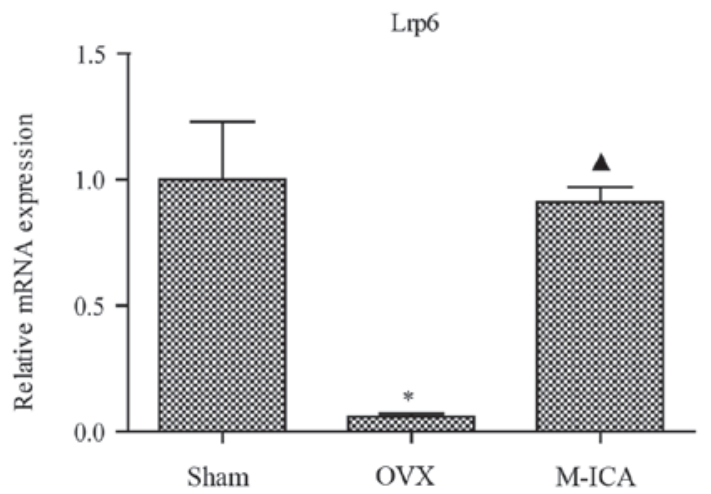

C

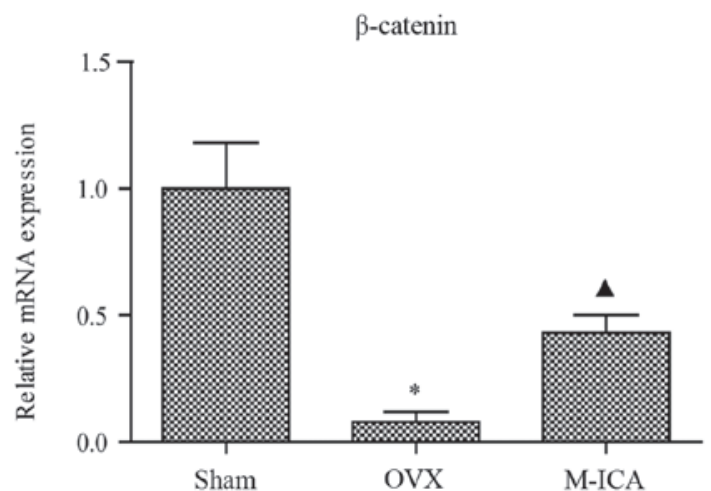

B

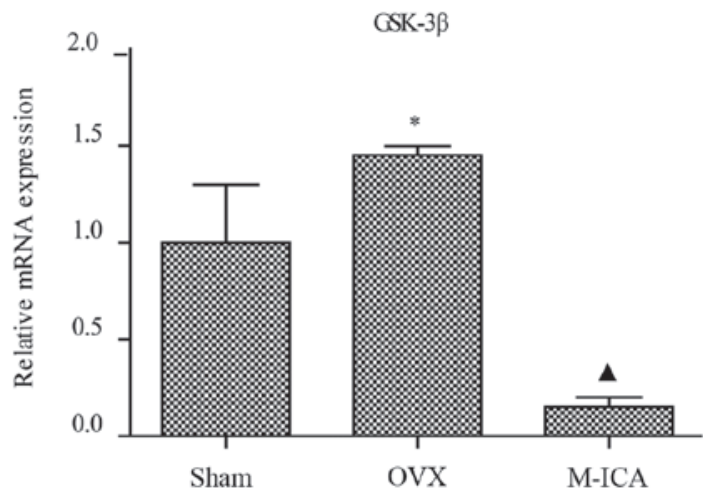

D

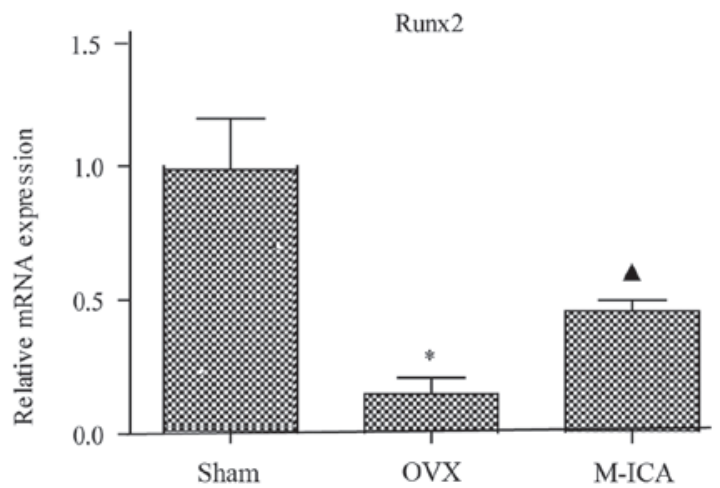

Figure 6. mRNA expression levels determined by reverse transcription-quantitative polymerase chain reaction. Expression levels of (A) Lrp6, (B) GSK-3 $\beta$,

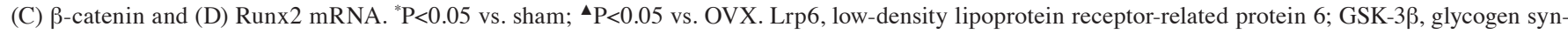
thase kinase-3 $\beta$; Runx2, runt-related transcription factor 2; OVX, ovariectomy; ICA, icariin; Sham, sham-operated; Positive, treated with alendronate sodium; L-ICA, low dose ICA (125 mg/kg/day); M-ICA, medium dose ICA (250 mg/kg/day); H-ICA, high dose ICA (500 mg/kg/day).

Effects of OVX and ICA on liver and kidney function. Following 12 weeks of treatment, BUN and ALT were significantly increased $(\mathrm{P}<0.05)$, as compared with the sham-operated group. As compared with the OVX group, M-ICA successfully restored BUN and ALT to normal levels $(\mathrm{P}<0.05)$, whereas $\mathrm{H}$-ICA significantly increased $\mathrm{Cr}(\mathrm{P}<0.05)$. Liver and kidney function index tests demonstrated the M-ICA group exhibited reduced liver and kidney damage, as compared with the OVX group (Table III).

Morphometric measurements. Morphological observations were quantitated by the histomorphometric analysis of longitudinal cross-sections collected $2 \mathrm{~cm}$ away from the proximal vertebra using micro-CT. Marked bone loss was observed 


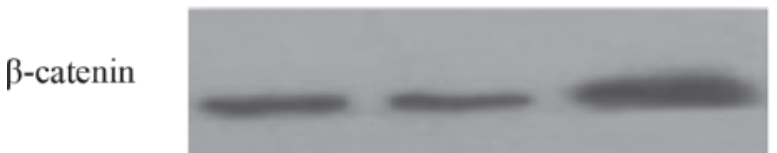

GAPDH

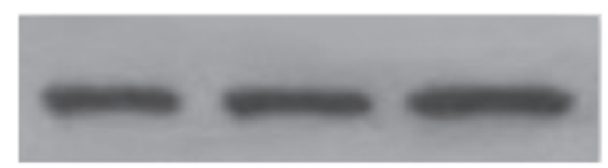

Sham-operated OVX M-ICA

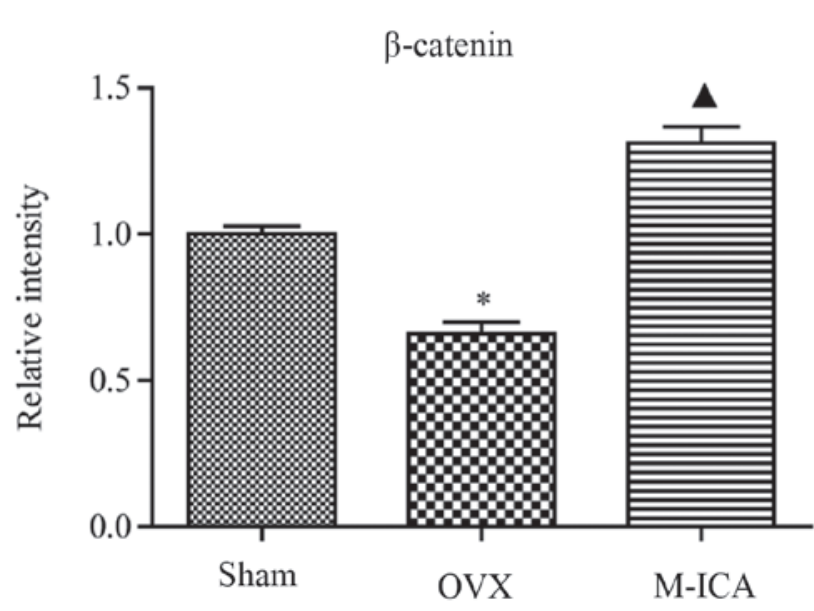

Figure 7. Western blotting results for $\beta$-catenin. ${ }^{\text {"P }}<0.05$ vs. sham; ${ }^{\mathbf{\Delta}} \mathrm{P}<0.05$ vs. OVX. GAPDH, glyceraldehyde 3-phosphate; OVX, ovariectomy; M-ICA, medium dose icariin $(250 \mathrm{mg} / \mathrm{kg} /$ day $)$.

in the OVX rats compared with the sham-operated group (Fig. 4A). This bone loss was accompanied by a significant reduction in Tb.Th and Tb.N together with a significant increase in Tb.Sp $(\mathrm{P}<0.01)$. Treatment with M-ICA normalized Tb.Th, Tb.N and Tb.Sp compared with the OVX group. Compared with the sham-operated group, in the positive group and the H ICA group, Tb.Th was significantly reduced $(\mathrm{P}<0.01)$ but Tb.Sp was significantly increased $(\mathrm{P}<0.05)$. In the L-ICA group, Tb.N decreased $(\mathrm{P}<0.05)$. In the L-ICA group, Tb.N decreased $(\mathrm{P}<0.05)$. Compared with the sham-operated group, the bone trabecula was looser in the OVX group. Following 12 weeks of treatment, as compared with the OVX group, the bone trabecula was more dense in the positive group and ICA groups, suggesting that ICA may recover bone trabecula to varying levels. M-ICA was most effective in comparison with the positive group (Fig. 4B).

Histopathology of femural bone. Histopathological changes in the femur were examined at the end of the 12-week study period. Representative histological sections of femur tissue were stained with H\&E. Tissue from the sham-operated group revealed normal compactness of the diaphysis and a competent trabecular meshwork (Fig. 5A). Tissue from the OVX group showed sparse, uniform thinning of the trabeculae, resulting in widened inter-trabecular spaces (Fig. 5B). In the positive group, the trabecular was significantly recovered (Fig. 5C). Tissues from the OVX-ICA groups exhibited significant restoration of structure, with mineralization. Uniform trabeculae with dense matrices and shaft sizes were also observed (Fig. 5D-F). Histomorphological parameters were within normal limits and complete restoration of the trabecular meshwork was observed. Bone from the M-ICA group (Fig. 5E) was essentially the same as normal bone.

Effect of ICA on expression of $\beta$-catenin, GSK-3 $\beta$ and Runx 2 and Lrp6 $m R N A$. The expression levels of $\beta$-catenin, Runx 2 and Lrp6 mRNA were reduced in the OVX animals compared with their respective levels in the sham-operated group $(\mathrm{P}<0.05$; Fig. 6). Levels of $\beta$-catenin, Runx 2 and Lrp6 mRNA in the M-ICA group were significantly increased in comparison with those in the OVX group $(\mathrm{P}<0.05)$. The levels of GSK-3 $\beta$ were significantly increased in the OVX group compared with those in the sham-operated group $(\mathrm{P}<0.05)$. Furthermore, treatment with M-ICA significantly reduced the expression of GSK-3 $\beta$ compared with that in the OVX group $(\mathrm{P}<0.05)$.

Effect of ICA on expression levels of $\beta$-catenin protein in OVX rats. As shown in Fig. 7, following bilateral removal of the ovaries, expression of $\beta$-catenin was significantly decreased $(\mathrm{P}<0.05)$. Treatment with M-ICA led to significantly increased levels of $\beta$-catenin compared with those in OVX rats $(\mathrm{P}<0.05)$.

\section{Discussion}

There has been much interest in the potential of phytoestrogen flavonoids as an alternative therapy for improving bone formation and for preventing or treating osteoporosis. ICA, a prenylated flavonol glycoside isolated from HEP, has been shown to prevent osteoporosis in late postmenopausal women (12) and has also been shown to have a strong estrogen-like activity (13).

The main index for evaluating the effect of treatments on postmenopausal osteoporosis (POP) is the increase in BMD. Estrogen deficiency, in both OVX rats and in postmenopausal women, can lead to a reduction in $\operatorname{BMD}(14,15)$. In the present study, treatment with ICA significantly improved BMD and effectively prevented the loss of bone mass caused by the OVX-induced lack of estrogen.

The bone changes observed in the OVX rats, most notably a decline in BMD, are similar to those in postmenopausal osteoporotic women. ICA treatment significantly increased BMD in the OVX rats and can effectively prevent bone loss and be effective in the treatment of osteoporosis in the present study.

Biomechanical testing showed that less force was needed to fracture the femur and vertebrae in OVX rats compared with sham-operated animals. Administration of ICA greatly improved the biomechanical properties, as demonstrated by the increased fracture force required to break the femur and vertebrae in OVX rats. Bone strength is associated with BMD, architecture, connectivity and mineralization (16). The results of the present study are consistent with those of this earlier study, in which ovariectomy resulted in reduced BMD as well as decreased biomechanical strength.

In the OVX rats, the bone tissue pathology and the morphology of bone microstructure changed markedly. Bone microstructure is an important factor affecting the quality of bone. Both bone microstructure and bone mass together 
determined bone strength. In OVX rats, the trabecular bone was clearly reduced in thickess and length, with a fine rod- or button-shaped morphology. Parts of the trabecular bone were destroyed, or even disappeared, and there were significant lesions in the secondary spongy bone area. The number of trabecular surface absorption pouches increased noticeably and there was a visible osteoclast (OC) reaction. Treatment with ICA reduced trabecular bone lesions, particularly in the secondary spongy areas, and reduced the number of bone resorption pouches and OCs. ICA can effectively improve BMD, increase trabecular bone numbers and thickness, and reduce their degree of separation.

The present study shows that an imbalance in the physiological processes of bone turnover, with loss of equilibrium between the activity of osteoblasts (OBs) and OC, contributes to the development of osteoporosis. Therapy at a molecular level should mainly aim to correct the imbalance between bone resorption and bone formation and thus protect skeletal integrity and reduce the risk of fractures. A previous study found that ICA strongly enhanced the differentiation and mineralization of $\mathrm{OB}$ in vitro (17), but the antiosteoporotic mechanism of ICA in vivo was not investigated. The present study indicates that ICA has the ability to correct the imbalance between bone resorption and bone formation in vivo.

Changes in serum biochemical indicators reflect the balance of bone metabolism in the body. These changes can be used to evaluate bone conversion, and to objectively measure bone resorption and bone formation. OPG and BGP are widely accepted as phenotypic markers of bone formation (18). OPG is a key cytokine secreted by $\mathrm{OB}$ that plays an important role in bone remodeling $(19,20)$. A negative correlation has been observed between OPG and BMD (21). BGP is regarded as a marker of bone turnover and serum levels of BGP have been shown to increase with increasing age (22). OPG produced by $\mathrm{OB}$ plays an essential role in the formation, differentiation and bone-resorbing activity of OC (23). It has been shown in the present study that OVX rats have reduced OPG activity compared with sham-operated animals. Administration of ICA to OVX rats upregulated OPG activity and downregulated BGP activity. In addition, the M-ICA dose did not cause liver or kidney damage.

ICA has been reported to enhance fracture healing (24), and treatment of OVX rats with ICA has been shown to ameliorate POP $(6,25,26)$. The main cause of osteoporosis is an imbalance between bone resorption and bone formation; healthy bones are required to maintain a dynamic balance between $\mathrm{OB}$ and $\mathrm{OC}$ for appropriate bone remodeling (27).

The Wnt receptors Lrp5/6 and Frizzled, when activated, promote phosphorylation of GSK-3 $\beta$ and block phosphorylation of $\beta$-catenin. Thus, the degradation of $\beta$-catenin is reduced and an increase in cytoplasmic and nuclear levels of $\beta$-catenin promotes pairing with the TCF/LEF family of transcription factors and activates the Wnt/ $\beta$-catenin signaling pathway $(28,29)$. Using RT-qPCR, the present study found that expression levels of Lrp6, $\beta$-catenin and Runx 2 mRNA decreased significantly in OVX rats, while the expression of GSK-3 $\beta$ mRNA increased significantly. Western blot analysis of the tibia showed that $\beta$-catenin expression also decreased significantly in OVX rats at the protein level. Treatment with ICA significantly increased the expression of Lrp6, $\beta$-catenin and Runx 2 mRNA and decreased the expression of GSK-3 $\beta$ mRNA. Upregulation of Lrp6 receptor should promote GSK- $3 \beta$ mRNA phosphorylation and increase the stability of intracellular $\beta$-catenin. Aggregation of $\beta$-catenin and transfer to the nucleus will activate the $\mathrm{Wnt} / \beta$-catenin signaling pathway. This then activates the target gene, Runx2, which promotes $\mathrm{OB}$ proliferation and differentiation and inhibits $\mathrm{OC}$ differentiation (30-32).

An ideal antiostoporotic agent should restore the balance between bone resorption and bone reconstruction, enhance bone strength and BMD, increase Th.N and reduce bone loss. The present in vivo study demonstrated that ICA effectively increased the BMD of the fourth and fifth vertebrae of the lumbar spine and femur in OVX rats. In addition, lumbar spinal Tb.N and Tb.Th were increased and Tb.Sp was reduced by ICA treatment. Bone strength was enhanced and bone loss was reduced. ICA was thus indicated to be an effective treatment for POP, that did not cause liver and kidney damage when used at an effective dosage. The action of ICA as an antiosteoporotic agent in OVX rats has been shown to involve upregulation of the expression of Lrp6 receptor mRNA and downregulation of GSK- $3 \beta$ mRNA. This promotes GSK- $3 \beta$ mRNA phosphorylation and increases the stability of intracellular $\beta$-catenin. The $\beta$-catenin aggregates and is transferred to the nucleus, where it activates the Wnt/ $\beta$-catenin signaling pathway. The downstream target gene, Runx 2 , is then activated, promoting OB proliferation and differentiation and inhibiting OC differentiation and bone resorption. This restores the balance between bone resorption and bone reconstruction, enhances bone strength and BMD, increases Th.N and reduces bone loss, all of which contribute to the treatment of osteoporosis.

\section{Acknowledgements}

The present study was supported by grants from the National Natural Science Foundation of China (grant nos. 81473509 and 81503384), the Cultivation for Scientific Research of First Affiliated Hospital of Jinan University (grant no. 2015103), Guangdong Provincial Natural Science Foundation (grant no. S2012040007531), the Cultivation and Innovation Fund for Scientific Research of Jinan University Youth Fund Project (grant no. 21612341), the Fundamental Research Funds for the Central Universities (grant no. 21614309), the Medical Scientific Research Foundation of Guangdong Province (grant no. B2014227) and the administration of Traditional Chinese Medicine of Guangdong Province (grant no. 20151178).

\section{References}

1. Kelly PJ: Is osteoporosis a genetically determined disease? $\mathrm{Br} \mathrm{J}$ Obstet Gynaecol 103 (Suppl 13): 20-27, 1996.

2. Rossouw JE, Anderson GL, Prentice RL, LaCroix AZ, Kooperberg C, Stefanick ML, Jackson RD, Beresford SA, Howard BV, Johnson KC, et al; Writing Group for the Women's Health Initiative Investigators: Risks and benefits of estrogen plus progestin in healthy postmenopausal women: Principal results from the Woman's Health Initiative randomized controlled trial. JAMA 288: 321-333, 2002.

3. Ma DF, Qin LQ, Wang PY and Katoh R: Soy isoflavone intake inhibits bone resorption and stimulates bone formation in menopausal women: Meta-analysis of randomized controlled trials. Eur J Clin Nutr 62: 155-161, 2008. 
4. Gao SQ, Fu DX and Zhang HM: Advances in the study on the treatment of osteoporosis with Herba Epimedii and its compound prescriptions. China J Chin Mater Med 24: 249-251, 1999.

5. Li C, Li Q, Mei Q and Lu T: Pharmacological effects and pharmacokinetic properties of icariin, the major bioactive component in Herba Epimedii. Life Sci 126: 57-68, 2015.

6. Nian H, Ma MH, Nian SS and Xu LL: Antiosteoporotic activity of icariin in ovariectomized rats. Phytomedicine 16: 320-326, 2009.

7. Mok SK, Chen WF, Lai WP, Leung PC, Wang XL, Yao XS and Wong MS: Icariin protects against bone loss induced by oestrogen deficiency and activates oestrogen receptor-dependent osteoblastic functions in UMR 106 cells. Br J Pharmacol 159: 939-949, 2010.

8. Pearson J, Dequeker J, Reeve J, Felsenberg D, Henley M, Bright J, Lunt M, Adams J, Diaz Curiel M and Galan F: Dual X-ray absorptiometry of the proximal femur: normal European values standardized with the European Spine Phantom. J Bone Miner Res 10: 315-324, 1995.

9. Fradinho MJ, Vale AC, Bernardes N, Caldeira RM, Vaz MF and Ferreira-Dias G: Biomechanical properties of the equine third metacarpal bone: In vivo quantitative ultrasonography versus ex vivo compression and bending techniques. J Equine Vet Sci 35: 198-205, 2015.

10. Effendy NM, Khamis MF and Shuid AN: Micro-CT assessments of potential anti-osteoporotic agents. Curr Drug Targets 14: 1542-1551, 2013

11. Noguchi A, Nakamura K, Sakata K, Sato-Fukuda N, Ishigaki T, Mano J, Takabatake R, Kitta K, Teshima R, Kondo K and Nishimaki-Mogami T: Development and interlaboratory validation of a simple screening method for genetically modified maize using a DeltaDeltaCq-based multiplex real-time PCR assay. Analytical Chem 88: 4285-4293, 2016.

12. Zhang G, Qin L and Shi Y: Epimedium-derived phytoestrogen flavonoids exert beneficial effect on preventing bone loss in late postmenopausal women: A 24-month randomized, double-blind and placebo-controlled trial. J Bone Miner Res 22: 1072-1079, 2007.

13. Ye HY and Lou YJ: Estrogenic effects of two derivatives of icariin on human breast cancer MCF-7 cells. Phytomedicine 12: 735-741, 2005.

14. Gambacciani M and Levancini M: Hormone replacement therapy and the prevention of postmenopausal osteoporosis. Przeglad Menopauzalny 13: 213-220, 2014.

15. Agata U, Park JH, Hattori S, Aikawa Y, Kakutani Y, Ezawa I, Akimoto $\mathrm{T}$ and $\mathrm{Omi}$ N: The impact of different amounts of calcium intake on bone mass and arterial calcification in ovariectomized rats. J Nutr Sci Vitaminol (Tokyo) 61: 391-399, 2015.

16. Einhorn TA: Bone strength: The bottom line. Calcif Tissue Int 51 333-339, 1992.

17. Ma HP, Ming LG, Ge BF, Zhai YK, Song P, Xian CJ and Chen KM: Icariin is more potent than genistein in promoting osteoblast differentiation and mineralization in vitro. J Cell Biochem 112: 916-923, 2011.

18. Evans DB, Bunning RA and Russell RG: The effects of recombinant human interleukin-1 beta on cellular proliferation and the production of prostaglandin E2, plasminogen activator, osteocalcin and alkaline phosphatase by osteoblast-like cells derived from human bone. Biochem Biophys Res Commun 166: 208-216, 1990.
19. Hofbauer LC, Khosla S, Dunstan CR, Lacey DL, Boyle WJ and Riggs BL: The roles of osteoprotegerin and osteoprotegerin ligand in the paracrine regulation of bone resorption. J Bone Miner Res 15: 2-12, 2000.

20. Simonet WS, Lacey DL, Dunstan CR, Kelley M, Chang MS Lüthy R, Nguyen HQ, Wooden S, Bennett L, Boone T, et al: Osteoprotegerin: A novel secreted protein involved in the regulation of bone density. Cell 89: 309-319, 1997.

21. Xie GQ, Lei DD, He HB, Gong JJ, Chen C, Chen P, Zhang H, Luo XH, Liao EY and Wu XP: Relationship between serum TGF- $\beta 1$, OPG levels and osteoporotic risk in native Chinese women. Clinica Chim Acta 423: 116-121, 2013.

22. Plantalech L, Guillaumont M, Vergnaud P, Leclercq M and Delmas PD: Impairment of gamma carboxylation of circulating osteocalcin (bone gla protein) in elderly women. J Bone Miner Res 6: 1211-1216, 1991.

23. Kwan Tat S, Padrines M, Théoleyre S, Heymann D and Fortun Y: IL-6, RANKL, TNF-alpha/IL-1: Interrelations in bone resorption pathophysiology. Cytokine Growth Factor Rev 15: 49-60, 2004.

24. Qin L, Zhang G, Hung WY, Shi Y, Leung K, Yeung HY and Leung P: Phytoestrogen-rich herb formula 'XLGB' prevents OVX-induced deterioration of musculoskeletal tissues at the hip in old rats. J Bone Miner Metab 23 (Suppl): S55-S61, 2005.

25. Qian G, Zhang X, Lu L, Wu X, Li S and Meng J: Regulation and of Cbfa1 expression by total flavonoids of Herba epimedii. Endocr J 53: 87-94, 2006

26. Mok SK, Chen WF, Lai WP, Leung PC, Wang XL, Yao XS and Wong MS: Icariin protects against bone loss induced by oestrogen deficiency and activates oestrogen receptor-dependent osteoblastic functions in UMR 106 cells. Br J Pharmacol 159: 939-949, 2010.

27. Teitelbaum SL: Bone resorption by osteoclasts. Science 289: 1504-1508, 2000

28. Ahmed Y, Nouri A and Wieschaus E: Drosophila Apc1 and Apc2 regulate Wingless transduction throughout development. Development 129: 1751-1762, 2002.

29. Liu C, Li Y, Semenov M, Han C, Baeg GH, Tan Y, Zhang Z, Lin $X$ and He X. Control of $\beta$-Catenin phosphorylation and degradation by a dual-kinase mechanism. Cell 108: 837-847, 2002 .

30. Cui Y, Niziolek PJ, MacDonald BT, Zylstra CR, Alenina N, Robinson DR, Zhong Z, Matthes S, Jacobsen CM, Conlon RA, et al: Lrp5 functions in bone to regulate bone mass. Nat Med 17: 684-691, 2011.

31. Yan Y, Tang D, Chen M, Huang J, Xie R, Jonason JH, Tan X, Hou W, Reynolds D, Hsu W, et al: Axin2 controls bone remodeling through the beta-Catenin-BMP signaling pathway in adult mice. J Cell Sci 122: 3566-3578, 2009.

32. Gambardella A, Nagaraju CK, O'Shea PJ, Mohanty ST, Kottam L, Pilling J, Sullivan M, Djerbi M, Koopmann W, Croucher PI and Bellantuono I. Glycogen synthase kinase $-3 \alpha / \beta$ inhibition promotes in vivo amplification of endogenous mesenchymal progenitors with osteogenic and adipogenic potential and their differentiation to the osteogenic lineage. J Bone Miner Res 26: 811-821, 2011. 